\title{
CLASSIFICAÇÃO CLIMÁTICA DE KÖPPEN E DE THORNTHWAITE E SUA APLICABILIDADE NA DETERMINAÇÃO DE ZONAS AGROCLIMÁticAS PARA O ESTAdo DE SÃO PAULO (1)
}

\author{
GLAUCO DE SOUZA ROLIM $\left({ }^{2 *}\right)$; MARCELO BENTO PAES DE CAMARGO $\left({ }^{3}\right)$; \\ DANIEL GROSSELI LANIA $\left({ }^{4}\right)$; JENER FERNANDO LEITE DE MORAES $\left({ }^{5}\right)$
}

\begin{abstract}
RESUMO
O clima pode ser entendido como as condições atmosféricas médias em uma certa região. Ele influencia diretamente a maioria das atividades humanas, em especial a agricultura na qual define o nível de produtividade agrícola, condicionado principalmente pela disponibilidade hídrica regional. Entretanto, os sistemas de classificações climáticas (SCC) são pouco utilizados no âmbito de estudos agrícolas pois, normalmente, considera-se sua escala de atuação muito abrangente. Dessa forma, valores médios mensais de temperatura máxima e mínima do ar de 27 estações termométricas e de 427 postos pluviométricos do Estado de São Paulo foram utilizados na atualização e melhoria do mapeamento dos SCC de Köppen modificado e de Thornthwaite, além da avaliação de potenciais aplicações em estudos de zoneamento agroclimáticos para o Estado de São Paulo. A utilização de dados de 427 localidades propiciou um mapeamento mais acurado do Estado pelas duas classificações. Avaliou-se a aplicabilidade das classificações em estudos agroclimáticos pela capacidade de separação dos climas pelos dois sistemas em relação aos elementos meteorológicos e componentes do balanço hídrico normal, com análises de dispersão dos dados, testes de separação de médias de Tukey e análises de cluster com dados independentes. O SCC de Köppen foi eficiente apenas na macroescala e com baixa capacidade de separação de tipos de climas em relação aos elementos meteorológicos (temperatura do ar, chuva) e elementos resultantes do balanço hídrico (evapotranspiração, deficiência e excedente hídrico). Conseqüentemente, não deve ser utilizado em estudos agrometeorológicos. O SCC de Thornthwaite permitiu separar eficientemente os climas na topoescala ou mesoescala, pois conseguiu resumir eficientemente as informações geradas por balanços hídricos normais, demonstrando capacidade para determinação de zonas agroclimáticas. Foram também feitas discussões sobre épocas de semeadura e qualidade de produtos agrícolas relacionadas com os SCC considerados.
\end{abstract}

Palavras-chave: agrometeorologia, zoneamento, análise de cluster.

\section{ABSTRACT \\ CLIMATIC CLASSIFICATION OF KÖPPEN AND THORNTHWAITE SISTEMS AND THEIR APPLICABILITY IN THE DETERMINATION OF AGROCLIMATIC ZONNING FOR THE STATE OF SÃO PAULO, BRAZIL}

\begin{abstract}
Climate can be understood as the mean atmospheric condition for a certain region. It affects directly most of the human activities, in special agriculture since it defines final yield levels, conditioned mainly by the regional water availability. However, the climatic classifications systems (SCC) are not often used for agricultural studies because usually their scales are considered very wide. Therefore monthly mean values of maximum and minimum air temperature of 27 termometric stations and 427 pluviometric
\end{abstract}

( $\left.{ }^{1}\right)$ Recebido para publicação em 26 de maio de de 2006 e aceito em 9 de maio de 2007.

$\left(^{2}\right)$ Centro de Ecofisiologia e Biofísica, Instituto Agronômico (IAC), Caixa Postal 28, 13020-902 Campinas (SP). E-mail: rolim@iac.sp.gov.br $\left({ }^{*}\right)$ Autor correspondente.

$\left({ }^{3}\right)$ Estudante, 2. ${ }^{\circ}$ ano Geografia, UNESP - Ourinhos (SP), Brasil.

(') Centro de Solos e Recursos Ambientais, Instituto Agronômico (IAC), Campinas (SP)

$\left({ }^{5}\right)$ Com bolsa de produtividade em pesquisa do CNPq. 
stations of State of São Paulo were utilized to update and improve the mapping of two SCC, Köppen modified and Thornthwaite, besides to evaluate their applicability for agroclimatic zonning for the State of São Paulo. The use of 427 localities resulted in amore accurate mapping of the State by both climatic classifications. Their applicability on agroclimatical studies was evaluated through the separation capacity of the climate types by two systems, in relation to the meteorological elements and components of normal water balance. It was used data dispersion analysis, Tukey's test and cluster analysis with independent data. The Köppen SCC showed to be efficient only for macroscale and low capacity of climate types separation in relation to meteorological elements (air temperature, rain) and data resulting from water balance (evapotranspiration, deficit and surplus). As a result this SCC should not be used in agrometeorological studies. On the other hand, Thornthwaite SCC efficiently separates the climate types in toposcale or mesoscale in view of the fact that it can efficiently resume generated informations by normal water balance, indicating ability to determine agroclimatical zones. Discussions were also made about sowing dates and final quality of agricultural products related to the two SCC considered question.

Key words: agrometeorology, zonning, cluster analysis.

\section{INTRODUÇÃO}

Os sistemas de classificações climáticas (SCC) são de grande importância, pois, analisam e definem os climas das diferentes regiões levando em consideração vários elementos climáticos ao mesmo tempo, facilitando a troca de informações e análises posteriores para diferentes objetivos.

Um dos SCC mais abrangentes é o de Köppen (KöPpen e GeIGER, 1928), que partindo do pressuposto que a vegetação natural é a melhor expressão do clima de uma região, desenvolveu um SCC ainda hoje largamente utilizado, em sua forma original ou com modificações. As modificações e críticas ao sistema são sempre relacionadas aos limites térmicos/hídricos dos tipos de climas determinados para diferentes regiões. A mais significativa adaptação deste SCC foi proposta por TREWARTHA (1954) que buscou determinar tipos de climas para os Estados Unidos e, de forma geral, simplificou o sistema de Köppen (CARTER E MATHER, 1966), tornando-o mais adaptável para uso em sistemas informatizados. Esta modificação de TREWARTHA (1954) foi motivada pela sugestão de FLOHN (1950) na qual os climas deveriam ser definidos de acordo com as massas de ar que atingem determinada região.

No Brasil, Setzer (1966) simplificou o método de Trewartha (1954) para determinar os tipos climáticos que ocorrem no Estado de São Paulo. Mais recentemente, o método de Köppen foi modificado para a Austrália (STERn et AL, 2005) com sucesso, mostrando também, que o sistema foi consistente e que pode auxiliar na determinação de locais para novas estações meteorológicas e, ainda, ser utilizado em trabalhos de mudanças climáticas se os dados meteorológicos forem de diferentes períodos históricos.

Burgos (1958) concluiu que classificações descritivas como a de Köppen trazem informações mais adaptadas a estudos geográficos e climatológicos do que agrometeorológicos, pois necessita de poucos parâmetros, simplificando demais situações complexas da relação do clima com a produtividade de culturas agrícolas. Entretanto, ainda poucos estudos trazem informações quantitativas da relação da classificação e sua aplicabilidade em estudos agrometeorológicos.

Já no SCC de Thornthwaite (ThornthwaITE, 1948), a planta não é vista como um instrumento de integração dos elementos climáticos, e sim, como simplesmente um meio físico pelo qual é possível transportar água do solo para a atmosfera. Dessa forma, um tipo de clima é definido como seco ou úmido relacionado às necessidades hídricas das plantas, ou seja, dependente de um balanço hídrico. Assim, este SCC é considerado um método mais refinado que o de Köppen para aplicações agrícolas (TREWARTHA, 1954). O mesmo autor, entretanto, critica que a evapotranspiração potencial utilizada no SCC é estimada em geral somente pela temperatura devido à falta de disponibilidade de medidas dos outros elementos meteorológicos. Dessa forma, isso faz com que o SCC de Thornthwaite não traga melhorias na definição dos climas em comparação à de Köppen, sendo ainda, um sistema muito mais complexo e de difícil entendimento.

Balling (1984), entretanto, verificou que o sistema de Thornthwaite trouxe uma sensibilidade muito maior na definição dos climas nos Estados Unidos e que a quantidade de elementos meteorológicos e de estações meteorológicas levados em consideração, define o grau de abrangência e de sensibilidade dos SCC. Sendo assim, este autor propõe a utilização de classificações baseadas em análise de cluster (TYRON, 1939) em que é possível a determinação numérica da similaridade entre os climas, considerando mais elementos meteorológicos ao mesmo tempo.

No Estado de São Paulo, devido ao seu relevo acidentado, posição geográfica e diferentes influências de massas de ar, constata-se grande 
diversidade climática, sendo complexo seu estudo. Segundo Pinto e Alfonsi (1972), o próprio tipo e desenvolvimento da agricultura paulista são reflexos desta complexidade, acarretando diferenças significativas em potenciais de produtividades agrícolas. SETZER (1966) realizou o último mapeamento dos tipos de clima para o Estado de São Paulo usando o SCC de Köppen.

O objetivo deste trabalho é a atualização e melhoria do mapeamento das classificações climáticas de Köppen modificada e de Thornthwaite para o Estado de São Paulo, decorrente de maior número de estações agrometeorológicas e avaliação das potenciais aplicações em estudos de zoneamentos agroclimáticos.

\section{MATERIAL E MÉTODOS}

Para as 19 estações do Instituto Agronômico (IAC) e oito do Instituto Nacional de Meteorologia (INMET), foram utilizados dados normais mensais de temperatura máxima e mínima do ar e de chuva. Já para os 400 postos do Departamento de Águas e Energia Elétrica do Estado de São Paulo (DAEE), que fazem mensurações somente de chuva, as temperaturas máximas e mínimas mensais foram estimadas a partir de coordenadas geográficas, com equações de PEDRo JúNIOR et al. (1991) para o planalto e LEPSH et al. (1990) para o litoral e Vale do Ribeira. Para os cálculos subseqüentes foi utilizada, então, a temperatura média mensal, estimada como a média aritmética entre a temperatura média máxima e a mínima mensal. O período mínimo de dados utilizados foi de 13 anos e o máximo de 30 anos, entre 1941 e 1990.

Utilizando esses dados, foi avaliado para cada localidade, o tipo de clima segundo a classificação climática de Köppen, simplificada por Setzer (1966) (Figura 1) e Thornthwaite (1948). Algumas modificações no método de Köppen foram feitas: Setzer (1966) foi ampliado para receber o tipo climático "Am" ,Tropical monçônico (chuvas excessivas durante o ano, porém com inverno seco), característico de regiões litorâneas do Estado e Vale do Ribeira.

Com a utilização do sistema de informação geográfica- SIG-ILWIS foi feita a interpolação espacial dos resultados de 427 localidades a partir do método de krigagem com modelos esféricos ajustados iterativamente no programa VARIOWIN 2.2 (Pannatier, 1996), buscando-se a minimização do coeficiente IGF (Indicative goodness of fit). Com esta interpolação foi possível, então, a obtenção de mapas climáticos do Estado de São Paulo para as duas classificações.
Para avaliar a aplicabilidade dos dois SCC em estudos de zoneamento agroclimático, buscou-se caracterizar a representatividade de cada elemento climático em cada tipo de clima. Esta representatividade é decorrente de dois fatores:

\begin{tabular}{|c|c|c|c|c|c|c|c|}
\hline \multicolumn{2}{|c|}{$\begin{array}{c}\text { Temperatura média } \\
\text { Normal } \\
\end{array}$} & \multirow{2}{*}{$\begin{array}{l}\text { Total de } \\
\text { chuva do } \\
\text { mês mais } \\
\text { seco (Pms) }\end{array}$} & \multirow{2}{*}{$\begin{array}{c}\text { Total de chuva anual } \\
\text { (P) }\end{array}$} & \multirow{2}{*}{\multicolumn{3}{|c|}{$\begin{array}{l}\text { Descrição do Tipo de Clima } \\
\text { segundo Köppen } \\
\text { (Climas Úmidos) }\end{array}$}} & \multirow[b]{2}{*}{ Símbolo } \\
\hline $\begin{array}{l}\text { do mês } \\
\text { mais frio }\end{array}$ & $\begin{array}{l}\text { do mês mais } \\
\text { quente }\end{array}$ & & & & & & \\
\hline \multirow{3}{*}{$>=18^{\circ} \mathrm{C}$} & \multirow{4}{*}{$>=22^{\circ} \mathrm{C}$} & $>=60 \mathrm{~mm}$ & & \multirow{2}{*}{\multicolumn{2}{|c|}{ TROPICAL }} & $\begin{array}{c}\text { sem estação } \\
\text { seca }\end{array}$ & Af \\
\hline & & \multirow{2}{*}{$<60 \mathrm{~mm}$} & $<2500-27,27$. Pms & & & \multirow{4}{*}{$\begin{array}{l}\text { Inverno } \\
\text { Seco }\end{array}$} & Aw \\
\hline & & & $>=2500-27,27 . P m s$ & & $\begin{array}{l}\text { ROPICAL com } \\
\text { ivas excessivas }\end{array}$ & & $\mathrm{Am}$ \\
\hline \multirow{4}{*}{$<18^{\circ} \mathrm{C}$} & & \multirow{2}{*}{$<30 \mathrm{~mm}$} & & \multicolumn{2}{|c|}{\begin{tabular}{|l|l|} 
& Quente \\
\end{tabular}} & & Cwa \\
\hline & $<22^{\circ} \mathrm{C}$ & & & $\frac{0}{0}$ & Temperado & & Cwb \\
\hline & $>=22^{\circ} \mathrm{C}$ & \multirow{2}{*}{$>=30 \mathrm{~mm}$} & & \multirow{2}{*}{ ๗ } & Quente & \multirow{2}{*}{$\begin{array}{l}\text { sem } \\
\text { estação } \\
\text { seca }\end{array}$} & Cfa \\
\hline & $<22^{\circ} \mathrm{C}$ & & & & Temperado & & Cfb \\
\hline
\end{tabular}

Figura 1. Chave para a classificação climática de Köppen simplificada por SETZER (1966), modificada para a inclusão do tipo climático "Am" (Tropical Monçônico).

1) a abrangência dos climas: quantificada pela distribuição dos valores observados dos elementos climáticos mensais: Chuva (P), Evapotranspiração Potencial (ETP), Evapotranspiração Real (ETR), Temperatura do ar (T), Deficiência Hídrica (DEF), Excedente Hídrico (EXC); anuais: Chuva (Panual), Acúmulo de Temperatura (AC-T). A quantificação dessas distribuições foi feita calculando-se os seguintes momentos estatísticos: média, mediana, $10^{\circ}$ e $2 .^{\circ}$ quartis, $50 \%, 90 \%, 99 \%$ de probabilidade de ocorrência e verificação dos valores extremos, representados por gráficos do tipo caixa (boxplot). A estimativa de ETP mensal foi feita por THORNTHWAITE (1948) e os componentes do balanço hídrico normal mensal (DEF, EXC, ETR) calculados por THORNTHWAITE E MATHER (1955) com capacidade de água disponível (CAD) igual a $125 \mathrm{~mm}$.

2) as diferenças entre os climas: para se estabelecer essas diferenças foi aplicado o teste de Tukey a $5 \%$ de significância em médias desbalanceadas (TUKEY, 1966), para cada elemento climático.

Além dessas análises para cada elemento climático, foi feita também, a análise de cluster "Agglomerative Hierarchical Clustering (AHC)", com método de otimização de WARD (1963), em função de todos os elementos climáticos mensais conjuntamente (P, ETP, ETR, DEF e EXC). Esta análise permite verificar a eficiência na separação dos climas pelos dois sistemas de classificação climática (SCC), pois compara os resultados destes sistemas com o conjunto independentemente dos elementos climáticos. 
Por fim, são discutidas algumas aplicações do SCC de Thornthwaite em estudos de zoneamento agroclimático relacionados à determinação de datas de semeadura e qualidade final de produtos agrícolas.

\section{RESULTADOS E DISCUSSÃO}

A grande densidade de pontos espaciais proporcionou boa acurácia na determinação dos tipos climáticos, tanto pelo método de Köppen (SETZER, 1966) modificado (Figura 2), como de Thornthwaite (Figura $3)$, em todas as regiões do Estado. Esta maior acurácia induz a modificações com relação à classificação de Köppen feita anteriormente por SETZER (1966). A maior diferença observada foi com relação ao clima "Cwa" que perdeu muitas áreas no planalto central em detrimento de outros tipos de clima. Esta perda decorre do fato que em muitas regiões a temperatura do mês mais frio foi maior que $18{ }^{\circ} \mathrm{C}$, favorecendo por sua vez os climas "Af", "Aw" e "Am". Nas regiões em que ocorriam somente climas "Af", agora na nova classificação há também climas " $\mathrm{Am}^{\text {", }}$ como no litoral, Vale do Ribeira e na região próxima ao norte do Estado do Paraná (esta última "Cfa", por Setzer,1966). Outras grandes mudanças foram o aumento do clima "Cfa" principalmente no nordeste do Estado, próximo ao Estado de Minas Gerais, e a redução considerável das áreas com clima " $\mathrm{Cfb}^{\prime}$ na faixa de latitude da cidade de São Paulo. Assim os climas de maior ocorrência são o "Aw" no norte e noroeste e o "Cfa" no centro do Estado. Os outros tipos de climas ocorrem no litoral e em regiões próximas do sul de Minas Gerais e Rio de Janeiro (Figura 2).

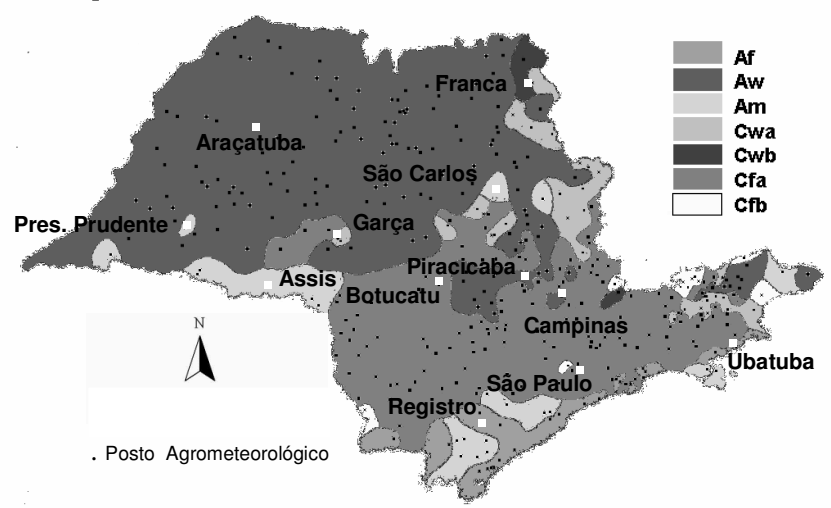

Figura 2. Classificação dos climas do Estado de São Paulo pelo sistema de Köppen (SETZER, 1966) modificado, com a utilização de dados agrometeorológicos de 427 localidades.

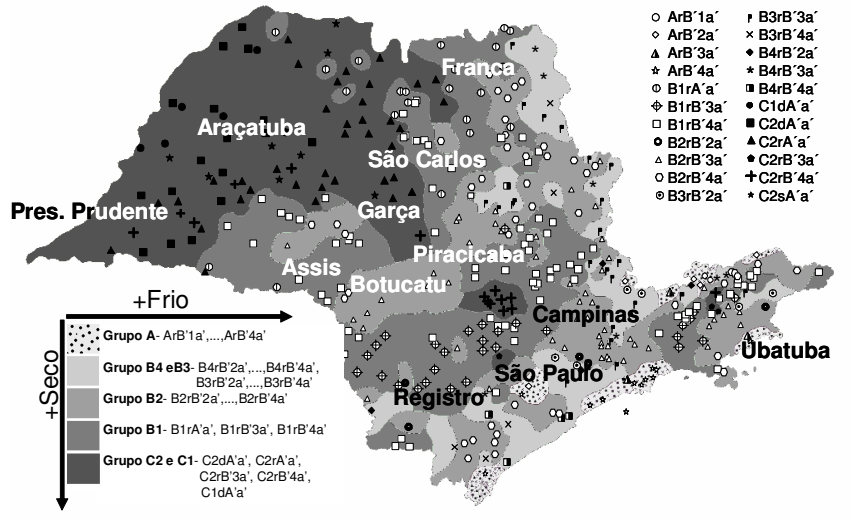

Figura 3. Classificação dos climas do Estado de São Paulo pelo sistema de THORNTHWAite (1948), com a utilização de dados agrometeorológicos de 427 localidades.

As grandes áreas homogêneas, principalmente com os climas "Aw" e "Cfa", indicam ser a classificação de Köppen muito abrangente, e seu uso mais apropriado na escala macroclimática. Mais detalhes serão tratados adiante. Dessa forma, pode-se verificar a influência dos fatores climáticos como de oceanalidade e continentalidade no condicionamento do clima litorâneo "Af" e o clima do interior do Estado "Aw", respectivamente, como também os climas "Cwa", "Cwb" que são influenciados pela elevada altitude.

Já a classificação de Thornthwaite, inédita para o Estado, demonstrou ser muito mais sensível aos montantes de chuva, temperatura e ao relevo das localidades, por resultar em um número maior de tipos de clima, totalizando 20 tipos. Esta sensibilidade é verificada no mapa (Figura 3) que não apresenta grandes áreas para cada tipo climático pois localidades bem próximas têm, muitas vezes, climas distintos. Os grupos de climas que mais ocorreram foram os do tipo ' $B$ ' (Úmidos), seguidos por ' $C$ ' (Subúmidos) e por fim 'A' (Superúmidos); os climas de maior ocorrência são: o $B^{\prime} 1 \mathrm{rB}^{\prime} 4 \mathrm{a}^{\prime}$, seguido por $\mathrm{C}^{\prime} \mathrm{rA}^{\prime} \mathrm{a}^{\prime}$ e por fim o clima B2rB'3 $\mathrm{a}^{\prime}$. Os climas B1rB' $4 \mathrm{a}^{\prime}$ e B2rB`3a' ocorrem na região central e o C2rA'a' está presente na Região Norte e Oeste do Estado.

Este maior número de tipos climáticos, em comparação aos de Köppen, indica ser a classificação de Thornthwaite útil na mesoescala ou topoescala, cujos efeitos da topografia têm efeito direto nos elementos climáticos e, por conseguinte, na atividade agrícola regional. Por exemplo, enquanto Köppen descreve como clima "Aw" o norte e oeste do Estado, Thornthwaite, na mesma área, define nove diferentes tipos de clima (Tabela 1). 
Tabela 1. Correspondência dos sistemas de classificação climática de Köppen modificado e de Thorhthwaite (1948) para 427 localidades do Estado de São Paulo

\begin{tabular}{|c|c|c|c|c|c|c|c|c|c|c|}
\hline Köppen & Thornthwaite & & & & & & & & & \\
\hline Af & $\mathrm{ArB}^{\prime} 4 \mathrm{a}^{\prime}$ & $\mathrm{B} 1 \mathrm{rB}^{\prime} 4 \mathrm{a}^{\prime}$ & $\mathrm{B} 2 \mathrm{rB}^{\prime} 4 \mathrm{a}^{\prime}$ & $\mathrm{B} 3 \mathrm{rB}^{\prime} 4 \mathrm{a}^{\prime}$ & $\mathrm{B} 4 \mathrm{rB}^{\prime} 4 \mathrm{a}^{\prime}$ & - & - & - & - & - \\
\hline $\mathrm{Am}$ & B1rA'a' & $\mathrm{B} 1 \mathrm{rB}^{\prime} 4 \mathrm{a}^{\prime}$ & $\mathrm{B} 2 \mathrm{rB}^{\prime} 4 \mathrm{a}^{\prime}$ & $\mathrm{B} 3 \mathrm{rB}^{\prime} 4 \mathrm{a}^{\prime}$ & $\mathrm{B} 4 \mathrm{rB}^{\prime} 4 \mathrm{a}^{\prime}$ & $\mathrm{C} 2 \mathrm{rA}^{\prime} \mathrm{a}^{\prime}$ & $\mathrm{C} 2 \mathrm{rB}^{\prime} 4 \mathrm{a}^{\prime}$ & - & - & - \\
\hline Aw & $\mathrm{C} 2 \mathrm{rA}^{\prime} \mathrm{a}^{\prime}$ & B3rB'3a' & $\mathrm{B} 2 \mathrm{rB}^{\prime} 4 \mathrm{a}^{\prime}$ & $\mathrm{B} 3 \mathrm{rB}^{\prime} 4 \mathrm{a}^{\prime}$ & B1rA'a' & $\mathrm{B} 1 \mathrm{rB}^{\prime} 4 \mathrm{a}^{\prime}$ & $\mathrm{C} 1 \mathrm{dA} \mathrm{A}^{\prime} \mathrm{a}^{\prime}$ & $\mathrm{B} 1 \mathrm{rB}^{\prime} 4 \mathrm{a}^{\prime}$ & $\mathrm{C} 2 \mathrm{rB}^{\prime} 4 \mathrm{a}^{\prime}$ & - \\
\hline $\mathrm{Cfa}$ & $\mathrm{ArB}^{\prime} 1 \mathrm{a}^{\prime}$ & $\operatorname{ArB}^{\prime} 2 a^{\prime}$ & $\mathrm{ArB}^{\prime} 3 \mathrm{a}^{\prime}$ & $\mathrm{ArB}^{\prime} 4 \mathrm{a}^{\prime}$ & $\mathrm{B} 1 \mathrm{rA}^{\prime} \mathrm{a}^{\prime}$ & $\mathrm{B} 1 \mathrm{rB} \mathrm{B}^{\prime} 3 \mathrm{a}^{\prime}$ & $\mathrm{B} 1 \mathrm{rB}^{\prime} 4 \mathrm{a}^{\prime}$ & $\mathrm{B} 2 \mathrm{rB}^{\prime} 2 \mathrm{a}^{\prime}$ & $\mathrm{B} 2 \mathrm{rB}^{\prime} 3 \mathrm{a}^{\prime}$ & $\mathrm{B} 2 \mathrm{rB}^{\prime} 4 \mathrm{a}^{\prime}$ \\
\hline B3rB'2a' & $\mathrm{B} 3 \mathrm{rB}^{\prime} 3 \mathrm{a}^{\prime}$ & $\mathrm{B} 3 \mathrm{rB}^{\prime} 4 \mathrm{a}^{\prime}$ & $\mathrm{B} 4 \mathrm{rB}^{\prime} 2 \mathrm{a}^{\prime}$ & $\mathrm{B} 4 \mathrm{rB}^{\prime} 3 \mathrm{a}^{\prime}$ & $\mathrm{B} 4 \mathrm{rB}^{\prime} 4 \mathrm{a}^{\prime}$ & $\mathrm{C} 1 \mathrm{dA} \mathrm{A}^{\prime} \mathrm{a}^{\prime}$ & $\mathrm{C} 2 \mathrm{rA}^{\prime} \mathrm{a}^{\prime}$ & $\mathrm{C} 2 \mathrm{rB}^{\prime} 3 \mathrm{a}^{\prime}$ & $\mathrm{C} 2 \mathrm{rB}^{\prime} 4 \mathrm{a}^{\prime}$ & - \\
\hline $\mathrm{Cfb}$ & $\mathrm{ArB}^{\prime} 1 \mathrm{a}^{\prime}$ & $\operatorname{ArB}^{\prime} 2 a^{\prime}$ & $\mathrm{B} 3 \mathrm{rB}^{\prime} 2 \mathrm{a}^{\prime}$ & $B 4 r B^{\prime} 2 a^{\prime}$ & - & - & - & - & - & - \\
\hline Cwa & $\mathrm{B} 1 \mathrm{rB}^{\prime} 3 \mathrm{a}^{\prime}$ & $\mathrm{B} 1 \mathrm{rB}^{\prime} 4 \mathrm{a}^{\prime}$ & $\mathrm{B} 2 \mathrm{rB}^{\prime} 2 \mathrm{a}^{\prime}$ & B2rB'3a' & B2 $\mathrm{rB}^{\prime} 4 \mathrm{a}^{\prime}$ & B3rB' $2 a^{\prime}$ & B3rB'3a' & B4rB'3a' & $\mathrm{C} 2 \mathrm{rB}^{\prime} 3 \mathrm{a}^{\prime}$ & $\mathrm{C} 2 \mathrm{rB}^{\prime} 4 \mathrm{a}^{\prime}$ \\
\hline $\mathrm{Cwb}$ & $\mathrm{B} 3 \mathrm{rB}^{\prime} 2 \mathrm{a}^{\prime}$ & $\mathrm{B} 4 \mathrm{rB}^{\prime} 2 \mathrm{a}^{\prime}$ & $\mathrm{B} 4 \mathrm{rB}^{\prime} 3 \mathrm{a}^{\prime}$ & - & - & - & - & - & - & - \\
\hline
\end{tabular}

Comparando-se os dois SCC (Tabela 1) verificou-se que não existiu equivalência entre o sistema de Köppen e o de Thornthwaite na determinação de tipos de clima para o Estado de São Paulo, pois, por exemplo, o clima B1rB' $4 a^{\prime}$ foi classificado nas mesmas localidades como "Af", "Am", "Aw", "Cfa" e "Cwa" pelo sistema de Köppen. Essa não-equivalência era esperada, pois cada um dos SCC tem pressupostos diferentes na avaliação de cada tipo climático, determinando tipos de clima, e como já comentado, em diferentes escalas climáticas.

Uma análise mais detalhada é necessária para se determinar a eficiência da utilização desses SCC em estudos de zoneamento agroclimático. Para tanto, a avaliação dos elementos climáticos como temperatura e chuva e, principalmente, valores resultantes do balanço hídrico das localidades, como ETP, ETR, DEF, EXC, fornecem subsídios para essas análises. As análises de ETR foram praticamente iguais às de ETP, para os dois tipos de classificação climática, razão pela qual não são aqui apresentadas.

O sistema de Köppen não conseguiu diferenciar de maneira clara os climas com relação aos valores de chuva no Estado de São Paulo (Figura 4.A e F), evidenciado, por exemplo, pela letra " $c$ " no teste de Tukey que ocorreu em quase todos os climas. Podese inferir somente que as localidades mais chuvosas apresentam clima tipo "Af". Nesse caso, a amplitude dos valores de chuva mensal observada varia de 60 $\mathrm{mm}$, nos meses mais secos, a $600 \mathrm{~mm}$, nos mais úmidos, com média por volta de $200 \mathrm{~mm}$ (Figura 4.A) e com total anual de até $4750 \mathrm{~mm}$ (limite superior a 99\% de probabilidade) (Figura 4.F). Outro clima úmido é o "Cfa" com amplitude mensal de $40 \mathrm{~mm}$ a $500 \mathrm{~mm}$, média de $60 \mathrm{~mm}$ e total anual de até $3.500 \mathrm{~mm}$. Apesar disso, com relação aos outros climas a distinção com relação à chuva mensal e anual não é significativa, especialmente em relação aos climas "Am", "Aw", "Cfb", "Cwa" e "Cwb".
O sistema de Köppen não conseguiu também diferenciar os climas do Estado de São Paulo com relação à temperatura média mensal (Figura 4.C) e acúmulo de temperatura anual (Figura 4.G), importantes para estimativas de duração de ciclos e subperíodos de culturas agrícolas. Verificou-se que o clima mais frio é o " $\mathrm{Cfb}$ ", depois em ordem crescente de temperatura, o "Cwb", "Cfa", "Cwa", "Af", "Am" e, por fim, "Aw". Na escala mensal (Figura $4 \mathrm{C}$ ), observa-se claramente o efeito do limite de $18{ }^{\circ} \mathrm{C}$ para a temperatura do mês mais frio usado para a determinação dos tipos de climas, principalmente em "Af", "Am" e "Aw". Entretanto, como os meses mais quentes possuem temperaturas muito próximas (26 $\left.{ }^{\circ} \mathrm{C}\right)$, não existem diferenças entre os climas, como também demonstrado pela análise de Tukey.

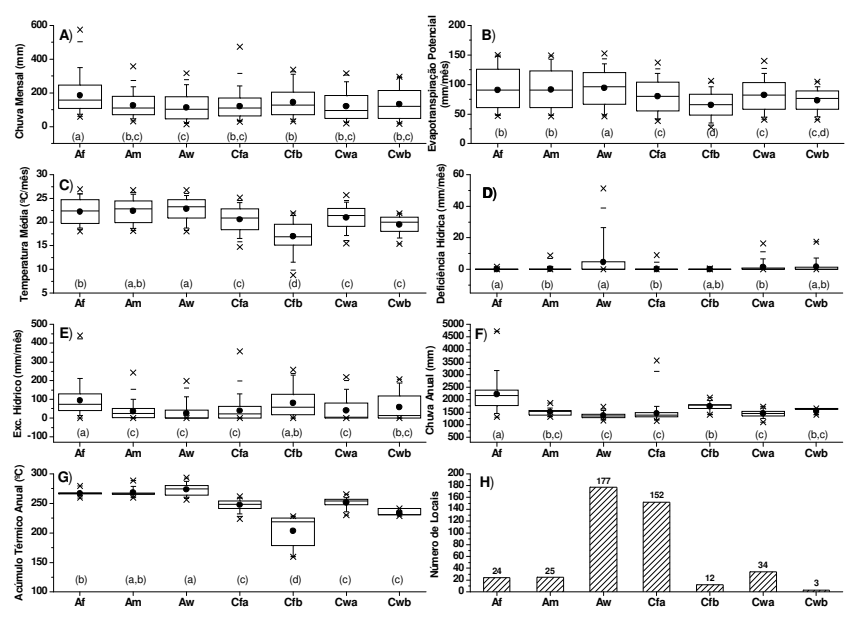

Figura 4. Distribuição de freqüências mensais de: A) Chuva; B) Evapotranspiração Potencial; C) Temperatura; D) Deficiência Hídrica; E) Excedente Hídrico, anuais; F) Chuva, G) Acúmulo Térmico; H) Número de locais, de localidades separadas por tipo de clima, determinados pelo sistema de Köppen modificado para o Estado de São Paulo. As letras entre parênteses são os resultados da comparação de médias do teste de Tukey a $5 \%$ de probabilidade. 
O sistema de Köppen também foi pouco sensível aos componentes do balanço hídrico. Com relação a DEF mensal (Figura 4.D), Köppen demonstra claramente que o clima " $\mathrm{Aw}$ " é potencialmente mais seco pela análise de dispersão dos dados. Entretanto, pelo teste de Tukey, constata-se que as médias entre os climas " $\mathrm{Aw}$ ", "Af", "Cfb" e "Cwb" não apresentam diferenças significativas (letra a). Já a respeito do EXC (Figura 4.E), verifica-se que o clima "Af" é o mais úmido, pois possui uma dispersão de dados de $0 \mathrm{a}$ $450 \mathrm{~mm} / \mathrm{mês}$. Para os outros climas, pelo teste de Tukey observa-se que não existem diferenças entre os climas "Am", "Aw","Cfa" e "Cwa". De forma geral, apesar da pouca diferença, avaliando-se tanto a DEF como o EXC mensal verifica-se que os climas em ordem crescente de umidade são: "Aw", "Cwb", "Cwa", "Am", "Cfa", "Cfb" e "Af",

Outro problema ocorre na abrangência do sistema, por exemplo, a DEF do clima "Aw" varia de $0 \mathrm{~mm} /$ mês até $40 \mathrm{~mm} /$ mês (Figura 4.D); já o segundo clima mais seco, o "Cwb" varia de $0 \mathrm{~mm}$ até $20 \mathrm{~mm}$, ou seja, metade do primeiro. Nesse caso, seria interessante a inclusão de um terceiro clima que atendesse uma faixa intermediária, para uma possível aplicação na escala topoclimática.

Deve-se lembrar que a definição de um tipo de clima leva em consideração vários elementos climáticos conjuntamente; dessa forma, a análise de quanto um clima é diferente de outro deve ser feita levando em consideração várias características que o diferem dos demais. Por exemplo, entre os climas que mais ocorrem no Estado: o "Aw" e "Cfa": no clima "Cfa" ocorre chuva mensalmente até $260 \mathrm{~mm}$ (Gráfico $4 \mathrm{~A}$ ) enquanto no " $\mathrm{Aw}$ " a chuva mensal é de até 240 $\mathrm{mm}$. O clima "Cfa", por sua vez, ocorre nos meses mais frios com média de $15^{\circ} \mathrm{C}$, enquanto no " $\mathrm{Aw}$ " a média fica por volta de $18{ }^{\circ} \mathrm{C}$ (Figura $4 \mathrm{C}$ ). Finalmente, a característica mais evidente que separa esses dois climas é que a DEF é muito maior no clima "Aw" chegando a $40 \mathrm{~mm}$ nos meses mais secos, enquanto no "Cfa" a DEF não ultrapassa 10 mm (Figura 4.D).

A análise de Cluster (AHC) permite uma avaliação da similaridade entre os climas levando em consideração vários elementos climáticos concomitantemente. No caso do sistema de Köppen (Figura 5.A) percebe-se que os climas " $\mathrm{Cfb}$ " e " $\mathrm{Af}$ ", "Aw" e "Am" e "Cfa" e "Cwa" são mais semelhantes nas condições do Estado de São Paulo.

Essa análise revelou também que o sistema de Köppen tem inconsistências, pois por exemplo, os climas " $\mathrm{Cfb}$ " e "Cwb", nos quais a diferença entre os dois seria somente em relação ao montante de chuva do mês mais seco $(30 \mathrm{~mm})$, na realidade, quando se leva em consideração os demais elementos climáticos conjuntamente, percebe-se que as diferenças são muito maiores, conforme figura 5.A, em que se verifica a grande distância euclidiana entre os dois climas (a máxima observada).

Por fim, resumidamente, percebe-se que a classificação de Köppen por se basear no dado de chuva anual e em alguns dados mensais (temperatura do mês mais quente, frio, e chuva do mês mais seco) e poucos tipos de clima, é muito abrangente atuando na escala macroclimática, não devendo ser utilizado em estudos agrometeorológicos como a determinação de zonas agroclimáticas.
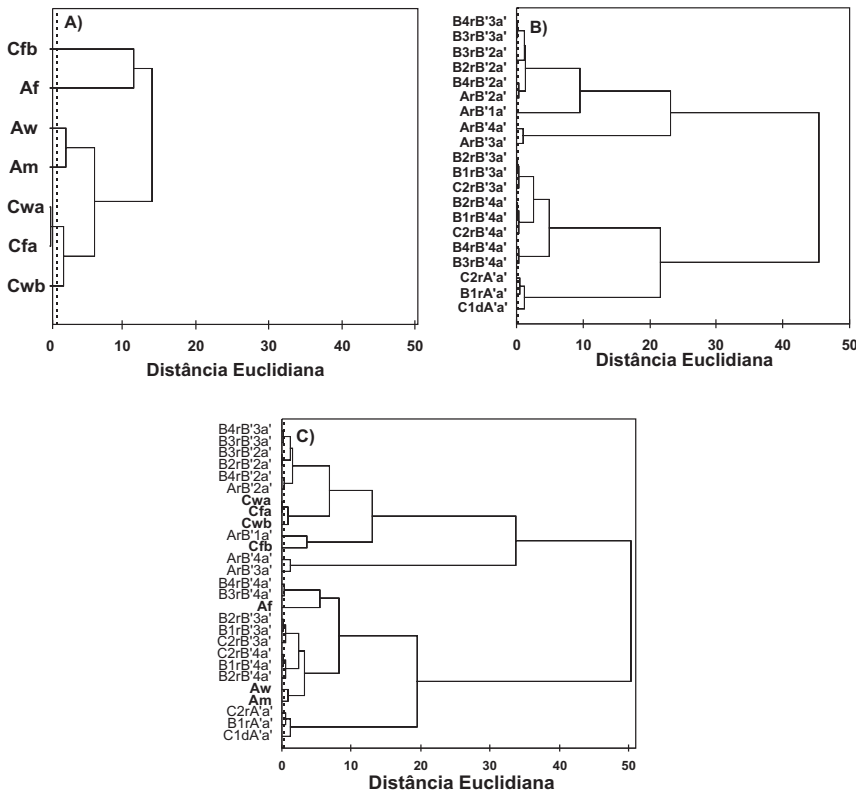

Figura 5. Dendrograma da análise de cluster obtida com seis indicadores (dados médios mensais de temperatura do ar, precipitação pluvial, evapotranspiração potencial e real, excedente e deficiência hídrica), com base no método de otimização de Ward (1963) para diferentes tipos de clima; A) Climas de Köppen; B) Climas de Thornthwaite; C) Comparação de Köppen e Thornthwaite.

Por sua vez, a classificação climática de Thornthwaite para o Estado de São Paulo, resultou em vinte tipos de clima (Figura 3), com resultados significativos quanto à separação dos climas em relação aos montantes de chuvas mensais (Figura 6.A) e anuais (Figura 6.F), importantes para a definição da produtividade final de culturas. Percebe-se que há boa definição dos climas tanto em relação à distribuição dos valores como em relação aos valores médios, demonstrado pelo teste de Tukey, em que se nota uma diferenciação gradual de médias nos diferentes climas.

Pode-se observar claramente que os climas " $C$ " são menos chuvosos (Figura 6.A), com média por volta de $100 \mathrm{~mm} /$ mês, com valores máximos absolutos ao 
redor de $200 \mathrm{~mm} / \mathrm{mês}$; os climas "B" são medianamente chuvosos, com média por volta de $125 \mathrm{~mm} / \mathrm{mês}$, com valores máximos absolutos ao redor de $300 \mathrm{~mm} / \mathrm{mês}$, e por fim, os climas " $\mathrm{A}$ " - os mais chuvosos, com média de $200 \mathrm{~mm} /$ mês, com máximos absolutos entre 300 a $600 \mathrm{~mm} / \mathrm{mês}$. Observa-se também, que os climas mais chuvosos foram o $\mathrm{ArB}^{\prime} 3 \mathrm{a}^{\prime}$ que chega a $3500 \mathrm{~mm} /$ ano (Figura 6.F), e ArB'4a' com valor máximo de $4500 \mathrm{~mm} /$ ano.
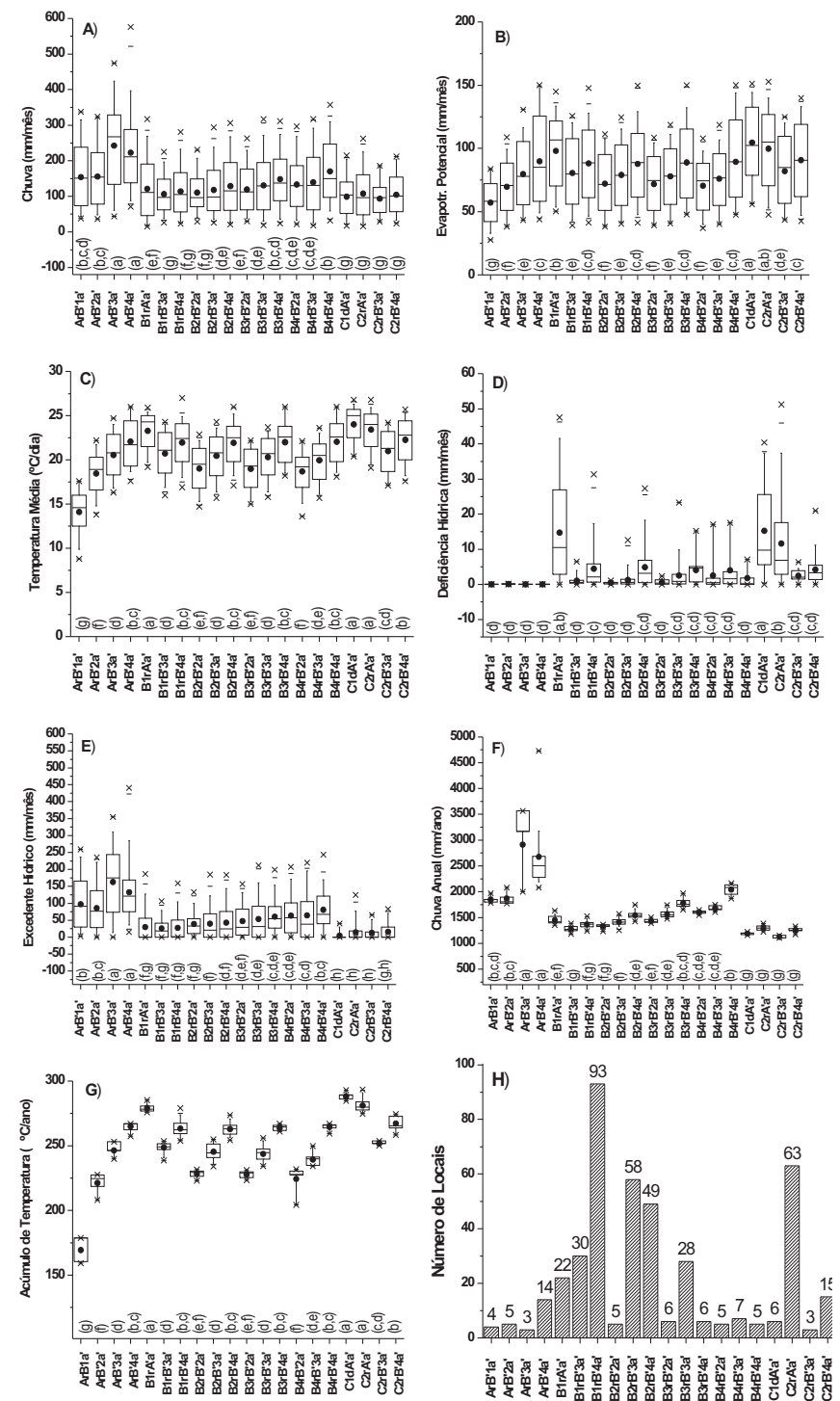

Figura 6. Distribuição de freqüências mensais de: A) Chuva, B) Evapotranspiração Potencial, C) Temperatura, D) Deficiência Hídrica, E) Excedente Hídrico; anuais: F) Chuva, G) Acúmulo Térmico; e H) Número de locais; de localidades separadas por tipo de clima, determinados pelo sistema de Thornthwaite para o estado de São Paulo. As letras entre parênteses são os resultados da comparação de médias do teste de Tukey a $5 \%$ de probabilidade.
Com relação aos aspectos de temperatura e de acúmulo de temperatura anual (Figura 6.C e G, respectivamente) verifica-se no sistema de Thornthwaite grande sensibilidade na diferenciação de tipos climáticos, verificado também pelo teste de Tukey. Os climas "C" são os mais quentes, com temperatura média anual de 24 ${ }^{\circ} \mathrm{C}$ e os " $\mathrm{A}$ " os mais frios, com a temperatura média anual ao redor de $19{ }^{\circ} \mathrm{C}$. Esse alto número de climas determinado pelo sistema atende de forma conveniente o zoneamento de grande número de culturas agrícolas, como por exemplo, a soja e a videira em temperaturas ótimas na faixa de 20 a $25{ }^{\circ} \mathrm{C}$; trigo e feijão em $15-20{ }^{\circ} \mathrm{C}$ e amendoim em 22-28 ${ }^{\circ} \mathrm{C}$ (Doorenbos e Kassam, 1979).

O SCC de Thornthwaite foi bastante sensível às variações de ETP (Figura 6 B) como também em relação aos elementos climáticos decorrentes do balanço hídrico normal (DEF, EXC), pois nos valores dos testes de Tukey notaram-se diferenças gradativas e crescentes nas diferenças das médias entre os climas. Percebe-se claramente que o sistema separa de forma eficiente, locais com diferenças de EXC (Figura 6 E), verificando-se que os climas " $C$ " são mais secos, com EXC médios de $25 \mathrm{~mm} /$ mês. Os climas "B" são medianamente secos e os climas " $\mathrm{A}$ " com maiores excedentes, chegando a valores extremos de $425 \mathrm{~mm} /$ mês nas condições do Estado. Houve sensibilidade no sistema, principalmente com relação ao fator térmico, que compõe a parte final da formulação do tipo de clima (PEREIRA ET AL., 2002), que influencia diretamente a ETP (Figura 6 B) e indiretamente a DEF (Figura 6 D). Dessa forma, os climas Megatérmicos (“...A'a' ") são os que possuem as maiores DEF, chegando a 50 $\mathrm{mm} / \mathrm{mês}$, seguidos pelos Mesotérmicos (“... $\mathrm{B}^{\prime} 4 \mathrm{a}^{\prime}$, $\ldots B^{\prime} 3 a^{\prime}, \ldots B^{\prime} 2 a^{\prime}, \ldots B^{\prime} 1 a^{\prime}$ “). Esta grande sensibilidade do sistema na separação de climas em relação aos componentes do balanço hídrico torna esse sistema útil na mesoescala, separando localidades próximas como, por exemplo, no planalto paulista: Ribeirão Preto $\left(\mathrm{B} 2 \mathrm{rB}^{\prime} 4 \mathrm{a}^{\prime}\right)$ e Serrana $\left(\mathrm{B} 1 \mathrm{rB}^{\prime} 4 \mathrm{a}^{\prime}\right)$, distantes apenas $25 \mathrm{~km}$.

Pela análise de cluster (Figura 5.B) observase, por exemplo, que no estado de São Paulo o clima $\mathrm{ArB}^{\prime} 2 \mathrm{a}^{\prime}$ é mais similar ao clima B4rB' $2 \mathrm{a}^{\prime}$ que ao clima ArB'1 '1a', levando-se em consideração a T, P, ETP, ETR, EXC e DEF concomitantemente. Desse modo, constatase que a parte final da simbologia do sistema de Thorthwaite, como já comentado, decorrente da temperatura e correspondência com os elementos calculados do balanço hídrico, tem grande peso na determinação dos climas. Pode-se, então, afirmar que o sistema de Thornthwaite consegue resumir eficientemente as informações geradas por balanços hídricos normais e/ou médios. 
Segundo Pereira et al. (2002), os resultados de um Balanço Hídrico Normal podem ser utilizados para o zoneamento agroclimático de uma região, determinação da demanda potencial de água das culturas irrigadas, de épocas de semeadura e conhecimento do regime hídrico. Conseqüentemente, devido aos resultados, pode-se afirmar que o SCC de Thornthwaite pode ser útil nas mesmas aplicações do balanço hídrico normal, por exemplo, para a determinação de melhores épocas de semeadura e plantio. A informação do tipo de clima pode também ser utilizada como uma camada de informação adicional em sistemas de informação geográfica para a elaboração de mapas de zoneamento agrometeorológico.
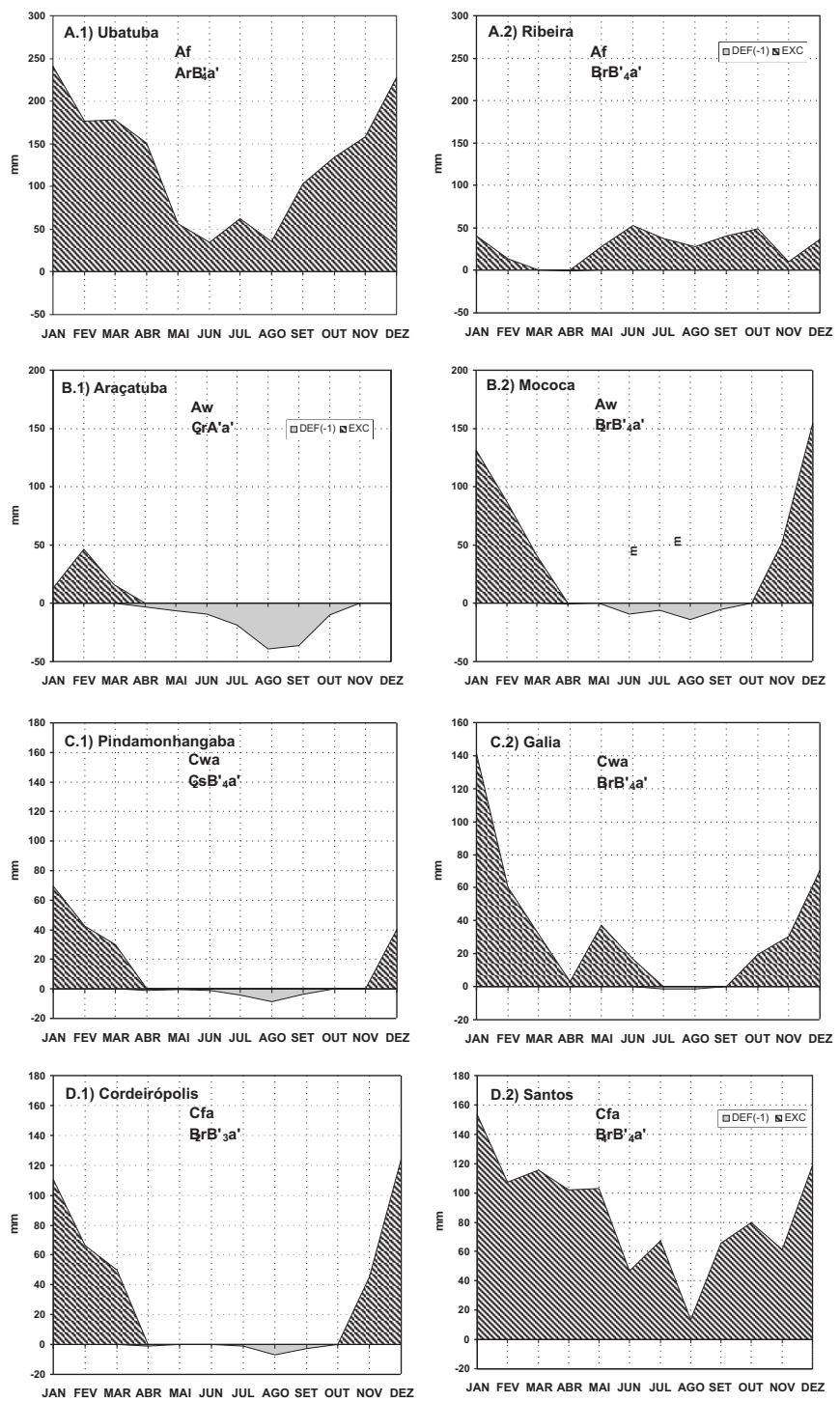

Figura 7. Extratos dos balanços hídricos normais mensais de (A.1)Ubatuba; (A.2) Ribeira; (B.1) Araçatuba; (B.2) Mococa; (C.1) Pindamonhangaba; (C.2) Gália; (D.1) Cordeirópolis e (D.2) Santos, com as respectivas classificações de Köppen e Thornthwaite.
Ilustrando essa possibilidade, na figura 7 são apresentados extratos de balanços hídricos de oito localidades nos quais se observa que o clima "C2rA'a'" (Araçatuba) tem um período de estiagem médio de abril a novembro, enquanto o clima “B2rB'4a'" (Mococa) o período é de abril a setembro, indicando que o período de semeadura pode ser, respectivamente, a partir de novembro e outubro. Outros exemplos podem ser visualizados na figura 7 , observando que o SCC de Thornthwaite resultou em diferentes climas para cada uma das localidades.

Observa-se também, a falta de sensibilidade do sistema de Köppen que classifica a localidade de Ubatuba (Figura 7 A-1) e Ribeira como "Af" (Figura 7 A-2), sendo o clima de Ubatuba muito mais semelhante ao de Santos (Cfa) (Figura 7.D-2) em termos de disponibilidade hídrica.

Esta análise de épocas de semeadura não pode ser feita com poucas localidades. Dessa forma, na figura 8, é apresentada a média de duração dos períodos mensais de EXC e DEF hídricas decorrentes da classificação de Thornthwaite para as 427 localidades do Estado de São Paulo. Verifica-se que o sistema consegue detectar uma ampla variedade de condições hídricas ao longo de um ano normal.

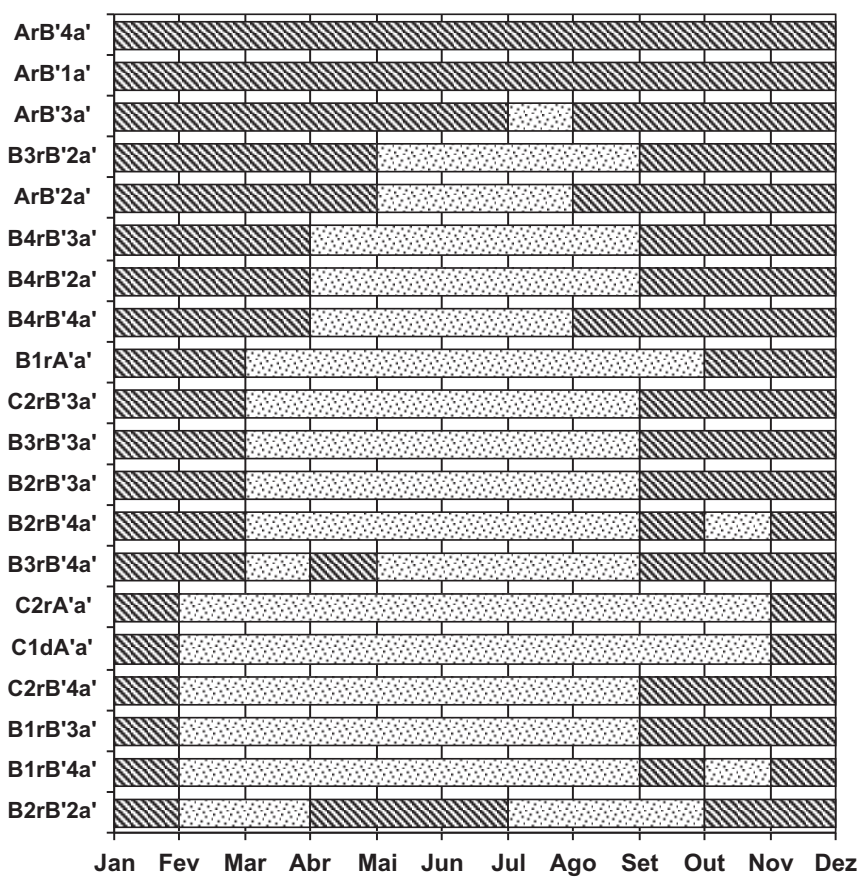

Figura 8. Valores médios de períodos de Excedente Hídrico mensal (barras escuras) e Deficiência Hídrica mensal (barras claras), calculados por Thornthwaite e Mather (1955) com CAD=125 mm para 427 localidades do Estado de São Paulo para cada tipo de clima, segundo o sistema de Thornthwaite (1948). 
O sistema de Thornthwaite pode ser útil também em outros aspectos no zoneamento agroclimático. Por exemplo, comparando-se o mapa final da classificação (Figura 3) com o mapa elaborado por ORTOLANI et al. 2000, que mostra regiões climáticas homogêneas na determinação da qualidade natural de bebida de café, observa-se que existem correspondências dos tipos de clima determinados conforme Thornthwaite e qualidade de café em São Paulo. Verifica-se que os climas B3 e B4, especialmente "B3rB'3a" e "B4rB'3a'" na Região de Franca (Alta Mogiana) correspondem às melhores condições, definindo a bebida tipo "Mole". Os climas B2 na região de Garça e Marília (B2rB'4a') conferem à bebida a denominação de "Dura adstringente". Por fim, a pior bebida, chamada de "Dura, pouco adstringente", característica de regiões mais secas como em Lins e Adamantina (ambos, C2rA'a'). Até mesmo o tipo de bebida "Riada", que é caracterizada pela perda de qualidade devido a chuvas na colheita, que ocorrem na região de Ourinhos, Avaré e Piraju são condicionadas por climas tipo B1.

Por fim, a determinação de zonas agroclimáticas se torna completa quando se utilizam coeficientes culturais específicos para cada cultura e/ou cultivar, entretanto, a utilização do sistema de classificação de Thornthwaite se torna interessante para: A) A extrapolação da zona agroecológica para outra área que não foi levada em consideração inicialmente no estudo; B) Pela facilidade de obtenção do tipo climático; C) Para culturas que não se possui ainda esses coeficientes culturais.

\section{CONCLUSÕES}

1. A utilização de maior número de estações meteorológicas melhorou a caracterização climática do Estado de São Paulo, segundo os sistemas de Köppen e de Thornthwaite.

2. O sistema de classificação climática de Köppen foi eficiente somente para a macroescala e, com baixa capacidade de separação de tipos de climas levando-se em consideração a temperatura do ar, a precipitação pluvial e elementos resultantes do balanço hídrico. Conseqüentemente, não deve ser utilizado em estudos agroclimáticos regionais como na determinação de zonas agrometeorológicas.

3. O sistema de Thornthwaite permitiu separar eficientemente os climas no Estado de São Paulo na topoescala, pois consegue resumir eficientemente as informações geradas por balanços hídricos normais, demonstrando, dessa forma, capacidade para determinação de zonas agroclimáticas.

\section{REFERÊNCIAS}

BALLING, R.C. Classification in climatology. In GAILE, G. L. AND WILLMOTT, C.J. (Ed). Spatial statistics and models. Hingham, MA: D. Reídel Publishing, 1984. p.81-108.

BURGOS, J.J. Agroclimatic classifications and representations: report of the applications value of climatic and agroclimatic classification for agricultural purposes. Varsovia: WMO, Comission for Agricultural Meteorology, 1958. (CaM II/Doc.18)

CARTER, D.B.; MATHER, J.R. Climatic classification for environmental biology. Elmer, NY: C.W. Thornthwaite Associates Laboratory of Climatology, 1966. 395p. (Publications in Climatology, v.19, n.4)

DOORENBOS, J.; KASSAM, A.H. Efeito da água no rendimento das culturas. Roma: FAO, 1979, 212p. (Irrigation \& Drainage papers, $\mathrm{n}^{\mathrm{o}} 33$ )

FLOHN, H. Neue anschauungen über die allgemeine zirkulation der atmosphäre und ihre klimatische bedeutung. Erdkunde, Bonn, v.4, p.141-162, 1950.

KÖPPEN, W.; GEIGER, R. Klimate der Erde. Gotha: Verlag Justus Perthes. 1928. Wall-map $150 \mathrm{~cm} \times 200 \mathrm{~cm}$.

LEPSH, I. F; SARAIVA, I. R; DONIZELI, P. L.; MARINHO, M. A.; SAKAI, E.; GUILLAUMON, J.R.; PFEIFER, R.M.; MATTOS, I.F.A.; ANDRADE, W.J.; SILVA, E.F. Macrozoneamento das terras da Região do Rio Ribeira de Iguape, SP. Campinas: Instituto Agronômico, 1990. 180p. (Boletim Científico, 19)

ORTOLANI, A.A.; CORTEZ, J.G.; PEDRO JUNIOR, M.J.; CAMARGO, M.B.P.; THOMAZIELLO, R.A.; ALFONSI, R.R. Clima e qualidade natural de bebida do café arábica no Estado de São Paulo. In: SIMPÓSIO DE PESQUISA DOS CAFÉS DO BRASIL, 1., 2000. Poços de Caldas. Anais... Brasília: EmbrapaCafé, 2000. v.I, p.662-664.

PANNATIER, Y. VARIOWIN: Software for Spatial Data Analysis in 2D. Springer-Verlag, 1996. 91p.

PEREIRA, A.P; ANGELOCCI, L.R.; SENTELHAS, P.C. Agrometeorologia: fundamentos e aplicações práticas. Lavras: Agropecuária, 2002. 478p.

PEDRO JÚNIOR, M.J.; MELLO M.H.A.; ORTOLANI, A.A.; ALFONSI, R.R., SENTELHAS, P.C. Estimativa das temperaturas médias mensais das máximas e das mínimas para o Estado de São Paulo. Campinas: Instituto Agronômico, 1991. 11p. (Boletim Técnico, 142)

SETZER, J. Atlas Climático e Ecológico do Estado de São Paulo. Comissão Interestadual da Bacia Paraná-Uruguai, 1966. 61p.

STERN,H; HOEDT,G; ERNST, J. Objective Classification of Australian Climates. Australia: Bureau of Meteorology.2005. Disponível em: http://www.bom.gov.au/climate/environ/ other/koppen_explain.shtml

THORNTHWAITE, C.W. An approach towards a rational classification of climate. Geographical Review, London, v.38, p.55-94, 1948. 
THORNTHWAITE, C.W.; MATHER, J.R. The Water Balance. Centerton, NJ: Drexel Institute of Technology, Laboratory of Climatology, 1955. 104p. (Publications in Climatology, v.8, n.1)

TREWARTHA, G.T. An Introduction to Climate. New York: McGraw-Hill, 1954. 402p.

TYRON, R. C. Cluster Analysis. Ann Arbor, MI: Edwards Brothers.1939. 422p.
TUKEY, J.W. The Problem of Multiple Comparisons, 9.ed. Princeton: Princeton University. 1953. 189p..

WARD, J. H. Jr. Hierarchical grouping to optimize an objective function. Journal of the American Statistical Association, Alexandria, v.58, p.236-244, 1963. 